\title{
Higher non-HDL-cholesterol to HDL- cholesterol ratio linked with increased nonalcoholic steatohepatitis
}

\author{
Dianhui Wang ${ }^{1}$, Ling Wang ${ }^{2}$, Zhanqing Wang ${ }^{3}$, Shihong Chen ${ }^{1}$, Yihong $\mathrm{Ni}^{1}$ and Dongqing Jiang ${ }^{1 *}$
}

\begin{abstract}
Background: Non-HDL-cholesterol to HDL-cholesterol (non-HDL-C/HDL-c) ratio is a feasible predictor for coronary heart disease, metabolic syndrome, and insulin resistance. Patients with nonalcoholic steatohepatitis (NASH) have an increased risk of developing cardiovascular problems and type 2 diabetes. However, the predictive role of non-HDLc/HDL-c ratio in NASH hasn't been investigated yet.
\end{abstract}

Methods: We conducted a retrospective cohort study. A total of 3489 eligible subjects were selected in the present study. Prevalence and characteristics of NASH were demonstrated. Conditional logistic regression was used to analyze the association between non-HDL-C/HDL-c ratio and risks of NASH. Associations between non-HDL-C/HDL-c ratio and serum aminotransferase levels were also investigated.

Results: The overall prevalence of NASH was 6.13\%, higher in male (6.89\%) than that in female (5.04\%). Interestingly, the prevalence of NASH showed a positive correlation with the elevation of non-HDL-c/HDL-c ratio (Pearson's Chi-squared test, linear trend $0.010, p<0.05$ ). The risk of NASH increased approximately 1.8 -fold among subjects with higher nonHDL-C/HDL-c ratio. After adjustment for confounding factors, higher non-HDL-c/HDL-c ratio was still associated with a 54. $4 \%$ increased risk of NASH. Male had higher risk of NASH than female when their non-HDL-c/HDL-c ratio increased. The risk of NASH in subjects with BMI more than 24 was 3 times higher than that in subjects with BMl less than 24. Every one unit increase in Non-HDL-c/HDL-c ratio was associated with 64.5\% increase in ALT/AST level $(p<0.05)$ after adjustment for confounding factors.

Conclusions: Our study provided strong evidence that subjects with higher non-HDL-c/HDL-c ratio had a higher risk of $\mathrm{NASH}$, which suggested that non-HDL-C/HDL-c ratio might be a feasible predictor for $\mathrm{NASH}$.

Keywords: Nonalcoholic steatohepatitis, Non-HDL-c/HDL-c ratio, Cohort study

\section{Background}

Non-alcoholic fatty liver disease (NAFLD) has emerged as the most common chronic liver disease worldwide in recent years, which was fueled by the increase in obesity and metabolic syndrome (MS) [1]. It ranges from fatty liver or hepatic steatosis to steatohepatitis with hepatic inflammation. About $5-20 \%$ of patients with fatty liver develop non-alcoholic steatohepatitis (NASH) during disease courses [2]. The morbidity and mortality in NASH patients are higher due to cardiovascular, cancer

\footnotetext{
* Correspondence: jiangdongqing@sina.com

${ }^{1}$ Department of Endocrinology and Metabolism, The Second Hospital of

Shandong University, Jinan 250033, Shandong, China

Full list of author information is available at the end of the article
}

and liver-related events including hepatocellular cancer compared with the general population [3]. Importantly, the initial stages of NASH are usually symptomless, thus with the advent of NASH symptoms, liver damages have progressed to cirrhosis and become inreversible [4]. Currently liver biopsy is the only way to diagnose NASH. However, it has limitations and is ineffective in many non-advanced cases. Therefore, it is imperative to identify valuable predictors for NASH in early stages, thus to prevent further exacerbation.

Non-high-density lipoprotein cholesterol (non-HDL-c) , which roughly equals to the total amount of lowdensity lipoprotein (LDL), very-low-density lipoprotein (VLDL), intermediate-density lipoprotein (IDL) and 
lipoprotein(a), is highlighted as a secondary target of lipid-lowering therapy [5]. UK Prospective Diabetes Study found that non-HDL-c/HDL-c ratio, rather than non-HDL-C, was a useful predictor for coronary heart disease (CHD) in type 2 diabetes patients [6]. Furthermore, this ratio was certified to be an effective predictor for CHD incidence in chronic kidney disease (CKD) patients [7], and an optimal predictor for MS and insulin resistance [8].

Since patients with NASH suffer an increased risk in developing cardiovascular problems, it is considerably valuable to further investigate the predictive role of nonHDL-c/HDL-c ratio in diagnosing NASH, which remains unknown. Therefore, we performed a cohort study to assess the association between the ratio of nonHDL-c to HDL-c and the risk of NASH. Our findings suggested that higher non-HDL-c/HDL-c ratio was positively associated with increased incidence of NASH. These findings highlight a pivotal role of non-HDL-c/ HDL-c ratio in the diagnosis of NASH.

\section{Methods}

\section{Ethics, consent and permissions}

This study was approved by the ethics committee of the Second Hospital of Shandong University with written informed consents from all participants.

\section{Participants}

Four thousand twenty-one individuals who received regular medical examination in our Hospital from 2015 to 2017 were retrospectively reviewed. Medical and surgical history for each subject was documented. The drinking histories were also recorded.

The following criteria were used for exclusion: (1) individuals with virus hepatitis; (2) alcohol abuse; (3) liver dysfunction due to cancer, autoimmune disease, genetic disease, or other disorders; (4) intake of drugs that influence serum lipids or liver function within the past 1 month; (5) hereditary hyperlipidemia. Finally, a total of 3489 eligible subjects (2061 males, 1428 females, with a mean age of $53.56 \pm 11.37$ years old) were selected and enrolled in the present study.

\section{Laboratory analyses}

Blood pressure, anthropometric measurements and blood specimens were obtained by well-trained clinical staff according to a standard protocol. Blood samples were collected from all participants between 8:00 $\mathrm{AM}$ and 10: 00 AM after a minimum 10-h fasting. All of the measurements were performed in the examination center that is affiliated to the Second Hospital of Shandong University.

Weight and height were measured in kilograms and centimeters, respectively, and BMI was calculated by dividing weight (kilograms) by the square of the height (square meters). Waist circumference was measured in centimeters. The blood pressure values were presented as the means of two measurements taken in the sitting position according to a standard protocol. The levels of alanine aminotransferase (ALT), aspartate aminotransferase (AST), fasting plasma glucose (FPG), total cholesterol (TC), triglycerides (TG), low-density lipoprotein cholesterol (LDL-c), high-density lipoprotein cholesterol $(\mathrm{HDL}-\mathrm{c})$, and Serum creatinine $(\mathrm{Cr})$ were determined using an Auto Biochemical Analyzer (MODULAR000GS; Roche, Basel, Switzerland). All participants were performed abdominal ultrasonic examination. The presence of NAFLD was detected by color ultrasonic diagnostic apparatus equipped with a $9 \mathrm{MHz}$ linear-array transducer (Toshiba Aplio 500 Ultrasound Scanner).

\section{Diagnostic criteria and definitions}

Fatty liver was diagnosed when a patient met any two of the following three ultrasonic criteria: liver and kidney echo discrepancy and presence of increased liver echogenicity (bright); unclear intrahepatic duct structure; liver far field echo decay [9]. Patients were excluded with potential cause of chronic liver disease, such as excessive alcohol consumption, hepatitis, or taking medications with a known association with fatty liver. The criterion for "nonalcoholic" is that daily alcohol consumption is lower than $20 \mathrm{~g}$ in women and $30 \mathrm{~g}$ in men. NASH is diagnosed as: 1) Serum ALT and/or AST levels were above $40 \mathrm{U} / \mathrm{L} ; 2)$ the elevation was sustained greater than 6 months; 3) NAFLD [10].

Hypertension was defined as a systolic blood pressure of $140 \mathrm{mmHg}$ or higher and a diastolic blood pressure of $90 \mathrm{mmHg}$ or higher, or being told by a doctor or health care professional on two or more different visits that he/ she had hypertension [11]. Diabetes mellitus (DM) was diagnosed if: 1) a self-reported previous diagnosis by health care professionals, 2) fasting plasma glucose $7.0 \mathrm{mmol} / \mathrm{L}$ or greater $(126 \mathrm{mg} / \mathrm{dL}), 3)$ a 2 -h plasma glucose in an oral glucose tolerance test $11.1 \mathrm{mmol} / \mathrm{L}$ or greater $(200 \mathrm{mg} /$ $\mathrm{dL})$, or 4) hemoglobin A1c $6.5 \%$ or greater [12].

\section{Statistical analysis}

Statistical analysis was performed using SPSS version 18. 0 (SPSS Inc). Continuous variables were presented as mean $\pm \mathrm{SD}$, and categorical variables were expressed as percentages (\%). Differences between groups were analyzed using one-way ANOVA for normally distributed continuous variables and chi-squared test for categorical variables. Univariate and multivariable logistic regression models were used to calculate odds ratios (OR) and 95\% confidence intervals (CI) for the associations between non-HDL-c/HDL-c ratio and NASH. Two sided $p$ values were calculated, and $p<0.05$ were considered to be statistically significant. 


\section{Results}

Baseline characteristics of the study population

Population composition and general characteristics were summarized in Table 1. Non-HDL-c/HDL-c ratios were stratified into three groups (group $\mathrm{A}: \leq 2.13$, group B: 2 . 13-2.89, and group C: $\geq 2.89$ ) defined by tertiles. Significant differences were detected among the 3 groups with respect to BMI, diastolic blood pressure (DBP), TC, TG, LDLc, FPG, Cr, ALT, AST, hypertension, and DM. Compared with group A, serum levels of lipid profiles, FPG, ALT, AST, and Cr were elevated in group C. In addition, increased BMI and DBP were more likely to accompany with hypertension and DM in subjects in group $C$ than that in group $\mathrm{A}$.

\section{The prevalence and distribution of NASH in the study population}

The overall prevalence of NASH was $6.13 \%$ (Fig. 1a). Interestingly, the prevalence of NASH in male (6.89\%) was higher than that in female $(5.04 \%)$. The prevalence of NASH increased linearly $(p<0.01)$ with age, and reached a peak in 50-59 group, then declined slightly (Fig. 1b).

\section{Progressive increase of NASH with the elevation of non- HDL-c/HDL-c ratio}

As shown in Fig. 2, the prevalence of NASH showed positively correlation with the elevation of non-HDL-c/
HDL-c ratio (Pearson's Chi-squared test, linear trend 0. $010, p<0.05)$. As was expected, subjects in group A had the lowest prevalence of NASH. While in group $\mathrm{C}$, subjects showed the highest prevalence of NASH, which was almost one time higher than that in group $\mathrm{A}$.

\section{High risk for NASH in subjects with higher non-HDL-c/ HDL-c ratio}

By using conditional logistic regression analysis, we found that the risk of $\mathrm{NASH}$ increased approximately 1.8-fold among subjects with higher non-HDL-c/ HDL-c ratio. After adjustment for confounding factors, subjects with higher non-HDL-c/HDL-c ratio were associated with $54.4 \%$ increased risk of $\mathrm{NASH}$ (Table 2). Compared with group A, the risk of NASH increased approximately 2.0-fold in subjects with nonHDL-c/HDL-c ratio levels greater than 2.89. These findings suggest that higher non-HDL-c/HDL-c ratio was highly positively associated with an increased risk of NASH.

Next, we analyzed the association between non-HDLc/HDL-c ratio and NASH after stratification by gender and BMI (Table 3). Male with increased non-HDL-c/ HDL-c ratio had higher risk of NASH compared with female. Dramatically, the risk of NASH in subjects with BMI more than 24 was 3 times higher than that in subjects with BMI less than 24.

Table 1 Baseline characteristics of the study population

\begin{tabular}{|c|c|c|c|c|}
\hline \multirow[t]{3}{*}{ Characteristic } & \multicolumn{3}{|c|}{ non-HDLC/HDLc ratio } & \multirow[t]{3}{*}{$P$ value } \\
\hline & $\mathrm{A}(\leq 2.13)$ & $\mathrm{B}(2.13-2.89)$ & $C(\geq 2.89)$ & \\
\hline & $(n=1162)$ & $(n=1163)$ & $(n=1163)$ & \\
\hline Age (yr), mean (SD) & $53.26(9.53)$ & $53.54(11.36)$ & $53.79(8.11)$ & 0.21 \\
\hline BMI $\left(\mathrm{kg} / \mathrm{m}^{2}\right)$, mean $(\mathrm{SD})$ & $23.72(3.10)$ & $24.32(3.20)$ & $25.68(3.38)$ & $<0.001$ \\
\hline TC (mmol/L), mean (SD) & $4.14(1.07)$ & $4.47(1.04)$ & $4.83(1.14)$ & $<0.001$ \\
\hline TG (mmol/L), median (IQR) & $1.10(1.10)$ & 1. $46(0.99)$ & $1.94(1.03)$ & $<0.001$ \\
\hline LDL-C (mmol/L), mean (SD) & $2.68(0.83)$ & $3.43(0.76)$ & $3.76(0.86)$ & $<0.001$ \\
\hline HDL-C (mmol/L), mean (SD) & $1.40(0.30)$ & $1.28(0.33)$ & $1.11(0.33)$ & 0.001 \\
\hline FPG (mmol/L), mean (SD) & $5.13(1.72)$ & $5.28(1.42)$ & $5.48(1.68)$ & $<0.001$ \\
\hline HbA1c (\%), mean (SD) & $5.26(1.14)$ & $5.31(1.06)$ & $6.04(1.03)$ & $<0.001$ \\
\hline SBP $(m m H g)$, mean (SD) & $124.19(20.70)$ & $124.74(18.53)$ & $125.75(20.79)$ & 0.36 \\
\hline DBP (mmHg), mean (SD) & $71.51(11.76)$ & $75.69(10.81)$ & $77.75(10.88)$ & $<0.001$ \\
\hline $\operatorname{ALT}(\mathrm{U} / \mathrm{L})$, mean (SD) & $15.54(11.42)$ & $19.94(10.15)$ & $27.66(10.79)$ & $<0.001$ \\
\hline AST (U/L), mean (SD) & $20.42(8.52)$ & $22.23(8.24)$ & $25.52(9.15)$ & $<0.001$ \\
\hline $\mathrm{Cr}(\mu \mathrm{mol} / \mathrm{L})$, mean $(\mathrm{SD})$ & $70.63(13.03)$ & 71.99 (12.08) & $73.46(10.27)$ & $<0.001$ \\
\hline DM, No. (\%) & $133(11.45)$ & $155(13.33)$ & $204(17.54)$ & $<0.001$ \\
\hline HT, No. (\%) & $468(40.28)$ & $489(42.05)$ & $526(45.23)$ & 0.01 \\
\hline
\end{tabular}

Continuous variables were compared by using one-way ANOVA and categorical variables by using the Chi-squared test. $\mathrm{P}<0.05$ was considered significant Abbreviations: $B M I$ body mass index, $T C$ total cholesterol, $T G$ triglyceride, $L D L-C$ low-density lipoprotein cholesterol, $H D L-C$ high-density lipoprotein cholesterol, FPG fasting plasma glucose, $H b A 1 c$ hemoglobin A1c, SBP systolic blood pressure, DBP diastolic blood pressure, $A L T$ alanine aminotransferase, $A S T$ aspartate aminotrasferase, $\mathrm{Cr}$ creatinine, DM diabetes mellitus, $H T$ hypertension 

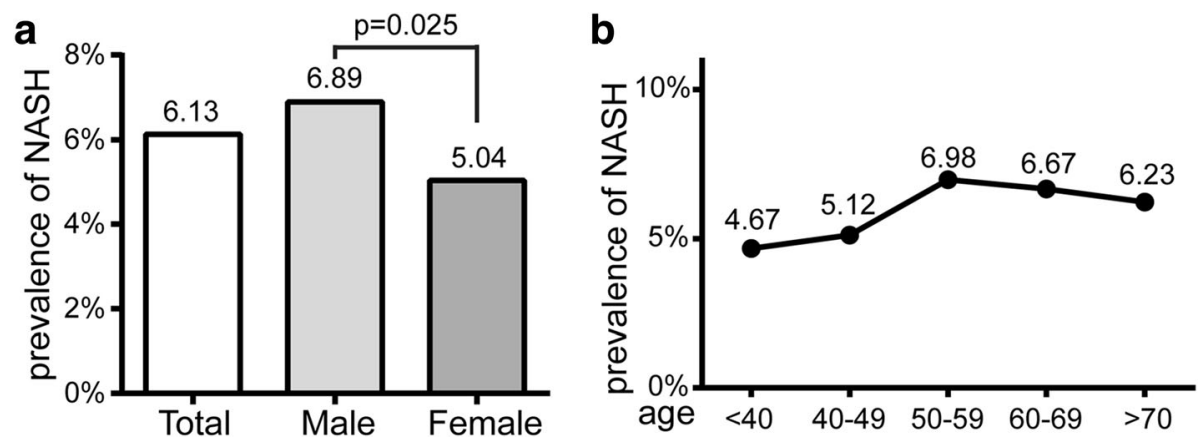

Fig. 1 The prevalence and distribution of NASH in the study population (a) and according to age group (b)

\section{Associations between non-HDL-c/HDL-c ratio and ALT/ AST}

Elevation of serum ALT and AST levels is a marker of abnormal liver function. Therefore, we further analyzed the associations between non-HDL-c/HDL-c ratio and serum ALT\&AST levels. Compared with group A, ALT and AST levels increased approximately 2.0-fold in subjects of group C. Importantly, after adjustment for confounding factors, the associations were even more significant (Table 4). By considering it as a continuous variable, we revealed that every one unit increase in Non-HDL-c/ HDL-c ratio was associated with $64.5 \%$ increase in ALT and AST levels $(p<0.001)$ after adjustment for confounding factors. These findings indicate that higher non-HDLc/HDL-c ratio was also a predictor for the elevation of serum ALT and AST levels in NASH.

\section{Discussion}

Our retrospective cohort study provided strong evidence for the first time that subjects with higher non-HDL-c/ HDL-C ratio had a higher risk of NASH. Our study indicated that the prevalence of NASH increased progressively with the elevation of non-HDL-c/HDL-c ratio.

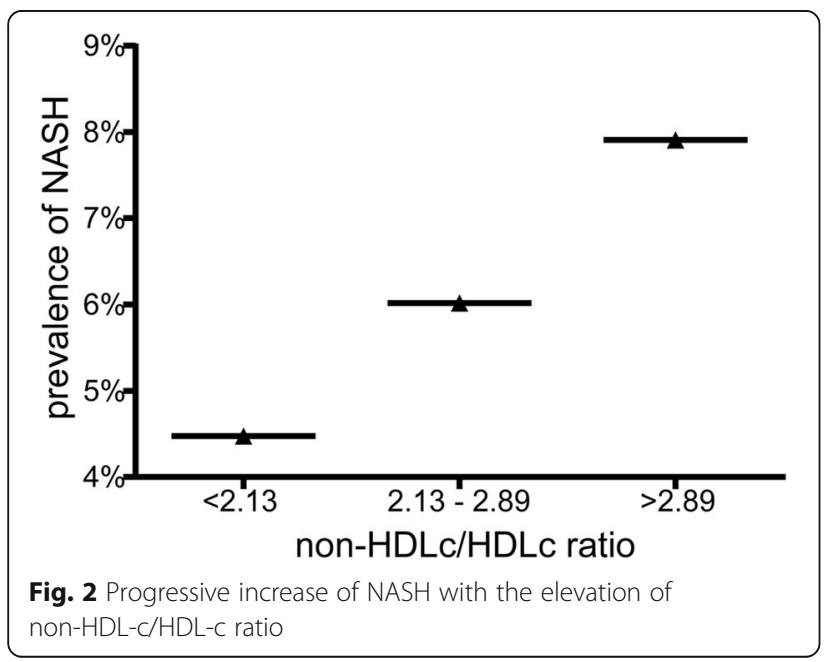

Higher non-HDL-c/HDL-c ratio was also a predictor for the elevation of serum ALT and AST levels in NASH. Our findings expanded the predictive role of non-HDL$\mathrm{c} / \mathrm{HDL}-\mathrm{c}$ ratio and suggested that increased non-HDL-c/ HDL-c ratio might facilitate the progression of liver injury in NAFLD.

Previous studies had demonstrated the correlation between dyslipidemia and liver disease. Obesity or dyslipidemia is one of the primary risk factors of NAFLD [13]. Many clinical trials showed that statin, a lipid-lowering drug, could substantially improve liver test indexes of patients $[14,15]$. The pathological process of dyslipidemia indicated that excessive cellular lipid accumulation occurred not only in adipose tissue but also in organs such as liver. Ectopic lipid accumulation in hepatocytes generated reactive oxygen species, resulting in lipid peroxidation, oxidative stress state and the releasing of several cytokines involving TNF- $\alpha$, TGF- $\beta$ and ILs [16]. Interestingly, Ciccone $\mathrm{MM}$ et al. reviewed and confirmed the role of NAFLD in increasing cardiovascular risk profile due to early impairment in vascular function and morphology [17]. The above complex inflammation circumstances led to hepatocytes apoptosis, collagen deposition, and abnormal proliferation of survived liver

Table 2 Conditional logistic regression analysis of non-HDLC/ $\mathrm{HDLC}$ ratio and risk for presence of NASH

\begin{tabular}{cccccc}
\hline & $B$ & SE & OR & $95 \% C l$ of OR & $P$ value \\
\hline non-HDLC/HDLc ratio & & & & & \\
Univariate Model & 0.456 & 0.095 & 1.778 & $1.309-1.982$ & $<0.001$ \\
Multivariate Model $^{a}$ & 0.283 & 0.143 & 1.544 & $1.023-1.756$ & 0.037 \\
Group A $(\leq 2.13)$ & & & 1 & & \\
Group B (2.13-2.89) & 0.223 & 0.155 & 1.356 & $1.076-1.528$ & $<0.001$ \\
Group C ( $\geq 2.89)$ & 0.682 & 0.173 & 1.963 & $1.409-2.776$ & $<0.001$
\end{tabular}

Data are coefficient (B), corresponding standard error, (SE), odds ratio (OR), 95\% confidence interval $(\mathrm{Cl})$ and significance ( $P$ value)

${ }^{a}$ Multivariate model was adjusted for body mass index, diastolic blood pressure, total cholesterol, triglyceride, low-density lipoprotein cholesterol, high-density lipoprotein cholesterol, hemoglobin A1c, alanine aminotransferase, aspartate aminotrasferase, creatinine, presence of diabetes, hypertension 
Table 3 Multivariate conditional logistic regression analysis of non-HDLC/HDLC ratio and risk for presence of NASH after stratification by gender and BMI

\begin{tabular}{clllll}
\hline Variables & B & SE & OR & $95 \%$ Cl of OR & $P$ value \\
\hline $\begin{array}{c}\text { Gender } \\
\text { Male }\end{array}$ & 0.716 & 0.151 & 2.047 & $1.522-2.753$ & $<0.001$ \\
Female & 0.465 & 0.062 & 1.592 & $1.410-1.797$ & $<0.001$ \\
BMl & & & & & \\
$\leq 24$ & 0.265 & 0.024 & 1.304 & $1.244-1.366$ & $<0.001$ \\
$>24$ & 0.874 & 0.175 & 4.316 & $2.137-7.523$ & $<0.001$ \\
\hline
\end{tabular}

Data are coefficient $(B)$, corresponding standard error, (SE), odds ratio (OR), 95\% confidence interval $(\mathrm{Cl})$ and significance $(P$ value). Multivariate model was adjusted for diastolic blood pressure, total cholesterol, triglyceride, low-density lipoprotein cholesterol, high-density lipoprotein cholesterol, hemoglobin A1c, alanine aminotransferase, aspartate aminotrasferase, creatinine, presence of diabetes, hypertension

cells, which would further result in chronic hepatocellular injury, liver cirrhosis and eventually liver cancer [18]. Although both clinical and laboratory researches proved the correlation between dyslipidemia and liver disease, the association between non-HDL-c/HDL-c ratio and the risk of NASH haven't been explored. Our results suggested that elevated non-HDL-c/HDL-c ratio may be a straightforward predictor for NASH and the progression of liver injury in NAFLD patients.

Serum aminotransferases such as ALT and aspartate AST can be considered as biochemical markers of liver dysfunction [19]. Elevations in ALT level are greater in patients with nonalcoholic steatohepatitis than in those with uncomplicated hepatic steatosis [20]. Moreover increased ALT level was associated with reduced insulin sensitivity, adiponectin and glucose tolerance as well as increased free fatty acids and triglycerides [21]. Elevated plasma ALT level was independently associated with increased risk of the metabolic syndrome in adults [22]. Meanwhile, elevated AST was observed in extensive tissue necrosis during myocardial infarction and in chronic liver diseases like

Table 4 Conditional logistic regression analysis of non-HDLC/ $\mathrm{HDLC}$ ratio and serum ALT/AST levels

\begin{tabular}{cccccc}
\hline & B & SE & OR & $95 \%$ Cl of OR & $P$ value \\
\hline non-HDLC/HDLc ratio & & & & & \\
Univariate Model & 0.265 & 0.024 & 1.304 & $1.244-1.366$ & $<0.001$ \\
Multivariate Model $^{a}$ & 0.468 & 0.035 & 1.645 & $1.359-2.536$ & $<0.001$ \\
Group A ( $\leq 2.13)$ & & & 1 & & \\
Group B (2.13-2.89) & 0.403 & 0.048 & 1.496 & $1.362-1.642$ & $<0.001$ \\
Group C ( $\geq 2.89)$ & 0.837 & 0.203 & 2.038 & $1.432-3.043$ & $<0.001$ \\
\hline
\end{tabular}

Data are coefficient (B), corresponding standard error, (SE), odds ratio (OR), 95\% confidence interval $(\mathrm{Cl})$ and significance ( $P$ value)

${ }^{a}$ Multivariate model was adjusted for body mass index, diastolic blood pressure, total cholesterol, triglyceride, low-density lipoprotein cholesterol, high-density lipoprotein cholesterol, hemoglobin A1c, alanine aminotransferase, aspartate aminotrasferase, creatinine, presence of diabetes, hypertension liver tissue degeneration and necrosis [19]. AST elevations often predominated in patients with cirrhosis and even in liver diseases typically with an increased ALT [23]. The positive association with elevation of ALT and AST levels highlighted the remarkable role of non-HDL-c/HDL-c ratio in predicting chronic liver injury.

NAFLD is currently the most common chronic liver disease worldwide. By 2025 it is estimated that 25 million Americans will have NASH, of whom 20\% are expected to develop cirrhosis or hepatocellular carcinoma [24]. It is expected that NASH will become the leading cause for liver transplant [25]. NAFLD/NASH is frequently described as the hepatic manifestation of metabolic syndrome [26]. As a result, patients with NASH carry a distinct set of comorbidities and risk factors that deserve special attentions [27]. Although hepatic biopsy is the gold standard to diagnose NASH, the procedure comes with a risk of major complications in $1-3 \%$ of patients and death in $0.01 \%$ of patients [28]. In this sense, noninvasively diagnostic tools was of urgent to detect the severity of steatosis or fibrosis with good accuracy $[29,30]$. To get non-HDL-c/HDL-c ratio was a simple and feasible tool to predict NASH. This approach could discriminate patients with increased cardiovascular risk in NAFLD patients. However, additional studies with larger populations and gold-standard hepatic biopsy are still needed to confirm our findings.

There were several limitations of this study. Since this was a cohort study in a single center, large and multiple center studies are still needed to verify the generalizability of the results. In addition, we could not conduct a liver biopsy study to confirm the diagnosis of NAFLD/NASH. Moreover, because we used a retrospective design, the causality between non-HDL-c/HDLc ratio and NASH cannot be fully established (i.e., we can only suggest an association). However, the strategy in this study was easily applied in large cohort study [9, 31]. A well-designed prospective research study will be necessary to address the correlations between non-HDL$\mathrm{c} / \mathrm{HDL}-\mathrm{c}$ ratio and NASH.

\section{Conclusions}

In summary, our results demonstrated that higher nonHDL-c/HDL-c ratio was associated with an increased risk of NASH. Our study provided a useful evidence for the primary prevention of individuals with NAFLD. Maintaining non-HDL-c/HDL-c ratio in an appropriate range, even by lifestyle interventions, might be an attractive and feasible approach to attenuate NAFLD/ NASH progression.

\section{Abbreviations}

ALT: Alanine aminotransferase; AST: Aspartate aminotransferase; $\mathrm{Cr}$ : Creatinine; FPG: Fasting plasma glucose; HDL-c: High-density lipoprotein cholesterol; LDL-c: Low-density lipoprotein cholesterol; NAFLD: Non-alcoholic 
fatty liver disease; NASH: Nonalcoholic steatohepatitis; TC: Total cholesterol; TG: Triglycerides

\section{Acknowledgements}

Not applicable.

\section{Funding}

Natural Science Foundation of China (81670753): The role of the funding body includes collection and analysis of the data.

Shandong Provincial Science and Technology Major Project

(2015ZDZX05005), Shandong Provincial Medical and Health Science and Technology Development Plan(2014WS0425), The Youth Talent Fund of The Second Hospital of Shandong University (2018YT33):

The role of these three funding bodies includes analysis of the data and writing of the manuscript.

\section{Availability of data and materials}

All data generated or analysed in this study are included in this published article.

\section{Authors' contributions}

DW and LW conducted the study, collected the data and drafted the manuscript. ZW analyzed and interpreted the data. YN and SC designed the study and revised the manuscript. DJ designed the study, revised the manuscript and gave final approval of the version to be published. All authors read and approved the final manuscript.

\section{Ethics approval and consent to participate}

The study was approved by the ethics committee of the Second Hospital of Shandong University.

\section{Consent for publication}

All of the participants agreed and provided written informed consent.

\section{Competing interests}

The authors declare that they have no competing interests.

\section{Publisher's Note}

Springer Nature remains neutral with regard to jurisdictional claims in published maps and institutional affiliations.

\section{Author details}

'Department of Endocrinology and Metabolism, The Second Hospital of Shandong University, Jinan 250033, Shandong, China. ${ }^{2}$ Department of Hospital-Acquired Infection Control, Laizhou City People's Hospital, Laizhou 261400, Shandong, China. ${ }^{3}$ Department of Emergency, Yantai Affiliated Hospital of Binzhou Medical University, Yantai 264100, Shandong, China.

Received: 16 January 2018 Accepted: 25 March 2018

\section{Published online: 03 April 2018}

\section{References}

1. Pappachan JM, et al. Non-alcoholic fatty liver disease: a clinical update. J Clin Transl Hepatol. 2017:5(4):384-93.

2. Bugianesi $E$, et al. Clinical update on non-alcoholic fatty liver disease and steatohepatitis. Ann Hepatol. 2008;7(2):157-60.

3. Sanyal AJ, et al. Challenges and opportunities in drug and biomarker development for nonalcoholic steatohepatitis: findings and recommendations from an American Association for the Study of Liver Diseases-U.S. Food and Drug Administration joint workshop. Hepatology. 2015;61(4):1392-405.

4. Di Bisceglie AM. Preventive strategies for chronic liver disease. Am Fam Physician. 2001;64(9):1515-6.

5. Expert Panel on Detection, E. and A. Treatment of High Blood Cholestero in. Executive summary of the third report of the National Cholesterol Education Program (NCEP) expert panel on detection, evaluation, and treatment of high blood cholesterol in adults (adult treatment panel III). JAMA. 2001;285(19):2486-97.

6. Lu W, et al. Non-HDL cholesterol as a predictor of cardiovascular disease in type 2 diabetes: the strong heart study. Diabetes Care. 2003;26(1):16-23.
7. Lamprea-Montealegre JA, et al. Chronic kidney disease, lipids and apolipoproteins, and coronary heart disease: the ARIC study. Atherosclerosis. 2014;234(1):42-6.

8. Kim SW, et al. Non-HDL-cholesterol/HDL-cholesterol is a better predictor of metabolic syndrome and insulin resistance than apolipoprotein B/ apolipoprotein A1. Int J Cardiol. 2013;168(3):2678-83.

9. Farrell GC, et al. Guidelines for the assessment and management of nonalcoholic fatty liver disease in the Asia-Pacific region: executive summary. J Gastroenterol Hepatol. 2007:22(6):775-7.

10. Watanabe $\mathrm{S}$, et al. Evidence-based clinical practice guidelines for nonalcoholic fatty liver disease/nonalcoholic steatohepatitis. J Gastroenterol. 2015;50(4):364-77.

11. Chobanian AV, et al. The seventh report of the joint National Committee on prevention, detection, evaluation, and treatment of high blood pressure: the JNC 7 report. JAMA. 2003;289(19):2560-72.

12. $\mathrm{Xu} Y$, et al. Prevalence and control of diabetes in Chinese adults. JAMA. 2013;310(9):948-59.

13. Loomba R, Sanyal AJ. The global NAFLD epidemic. Nat Rev Gastroenterol Hepatol. 2013;10(11):686-90.

14. Athyros VG, et al. Safety and efficacy of long-term statin treatment for cardiovascular events in patients with coronary heart disease and abnormal liver tests in the Greek atorvastatin and coronary heart disease evaluation (GREACE) study: a post-hoc analysis. Lancet. 2010;376(9756):1916-22.

15. Athyros VG, et al. Statin-based treatment for cardiovascular risk and nonalcoholic fatty liver disease. Killing two birds with one stone? Ann Med. 2011:43(3):167-71.

16. Reddy JK, Rao MS. Lipid metabolism and liver inflammation. II. Fatty liver disease and fatty acid oxidation. Am J Physiol Gastrointest Liver Physiol. 2006;290(5):G852-8

17. Ciccone MM, et al. Inflammatory bowel disease, liver diseases and endothelial function: is there a linkage? J Cardiovasc Med (Hagerstown), 2015;16(1):11-21.

18. Luedde T, et al. Deletion of NEMO/IKKgamma in liver parenchymal cells causes steatohepatitis and hepatocellular carcinoma. Cancer Cell. 2007; 11(2):119-32.

19. Gowda S, et al. A review on laboratory liver function tests. Pan Afr Med J. 2009;3:17.

20. Sheth SG, Gordon FD, Chopra S. Nonalcoholic steatohepatitis. Ann Intern Med. 1997:126(2):137-45.

21. Burgert TS, et al. Alanine aminotransferase levels and fatty liver in childhood obesity: associations with insulin resistance, adiponectin, and visceral fat. J Clin Endocrinol Metab. 2006:91(11):4287-94.

22. Shen $\mathrm{YH}$, et al. Bright liver and alanine aminotransferase are associated with metabolic syndrome in adults. Obes Res. 2005:13(7):1238-45.

23. Green RM, Flamm S. AGA technical review on the evaluation of liver chemistry tests. Gastroenterology. 2002;123(4):1367-84.

24. Burke A, Lucey MR. Non-alcoholic fatty liver disease, non-alcoholic steatohepatitis and orthotopic liver transplantation. Am J Transplant. 2004, 4(5):686-93.

25. Ong JP, Younossi ZM. Epidemiology and natural history of NAFLD and NASH. Clin Liver Dis. 2007:11(1):1-16. vii

26. Flemming $\mathrm{JA}$, et al. Reduction in liver transplant wait-listing in the era of direct-acting antiviral therapy. Hepatology. 2017;65(3):804-12

27. Carter D, Dieterich DT, Chang C. Nonalcoholic fatty liver disease/ nonalcoholic steatohepatitis in liver transplantation. Clin Liver Dis. 2018, 22(1):213-27.

28. Bravo AA, Sheth SG, Chopra S. Liver biopsy. N Engl J Med. 2001;344(7):495-500.

29. McPherson S, et al. Simple non-invasive fibrosis scoring systems can reliably exclude advanced fibrosis in patients with non-alcoholic fatty liver disease. Gut. 2010:59(9):1265-9.

30. Roitberg GE, et al. Non-invasive techniques in diagnosis of steatosis and fibrosis in nonalcoholic fatty liver disease. Eksp Klin Gastroenterol. 2013;(7): p. 3-9.

31. Watanabe $\mathrm{S}$, et al. Evidence-based clinical practice guidelines for nonalcoholic fatty liver disease/nonalcoholic steatohepatitis. Hepatol Res. 2015:45(4):363-77. 